\title{
Endometriosis clinical guidance during the COVID-19 pandemic
}

\author{
Mathew Leonardi ${ }^{1}$, Andrew Horne ${ }^{2}$, Mike Armour ${ }^{3}$, Stacey Missmer ${ }^{4}$, Horace Roman ${ }^{5}$, Luk \\ Rombauts $^{6}$, Lone Hummelshoj ${ }^{7}$, Arnaud Wattiez ${ }^{8}$, George Condous ${ }^{1}$, and Neil Johnson ${ }^{9}$ \\ ${ }^{1}$ Nepean Hospital \\ ${ }^{2}$ University of Edinburgh \\ ${ }^{3}$ Western Sydney University \\ ${ }^{4}$ Harvard University T H Chan School of Public Health \\ ${ }^{5}$ Clinique Tivoli-Ducos \\ ${ }^{6}$ Monash Medical School \\ ${ }^{7}$ Endometriosis.org \\ ${ }^{8}$ IRCAD, University Hospitals Strasbourg \\ ${ }^{9}$ Neil Johnson Gynaecology Clinic
}

April 28, 2020

The COVID-19 pandemic has led to a dramatic shift in the clinical practice of women's health and routine care for endometriosis has been severely disrupted. Endometriosis is defined as an inflammatory disease characterised by lesions of endometrial-like tissue outside the uterus that is associated with pelvic pain and/or infertility. ${ }^{1}$ It affects approximately $10 \%$ of reproductive age women worldwide, is diagnosed by surgically visualisation or by radiological imaging, and is treated with hormone treatments or by laparoscopic removal of lesions. ${ }^{2,3}$

Under the guidance of international gynaecological organisations ${ }^{4-6}$, many centres have temporarily ceased offering outpatient appointments, diagnostic imaging for non-acute pelvic pain, surgery for endometriosis, and fertility treatments. This means that endometriosis sufferers are be feeling particularly vulnerable and that resultant stress and anxiety may contribute to a worsening of symptoms. The pandemic poses several important questions for healthcare providers on how best to deliver care with these restrictions. Herein, we present clinical guidance on the management of endometriosis during the COVID-19 pandemic (Box 1).

Are endometriosis patients a high-risk population?

To date, there is no evidence that women with endometriosis are at increased risk of COVID-19. ${ }^{7}$ However, women with endometriosis within the chest cavity (in the lungs or on the diaphragm), referred to as 'thoracic endometriosis', may be more at risk. Similarly, there is no evidence that COVID-19 will hasten the progression/development of endometriosis. Nonetheless, the pandemic will likely contribute to a reduction in quality of life secondary to a delay in diagnosis and/or the treatment of endometriosis owing to the temporary closure of outpatient services, (including complementary therapies), postponement of planned surgical or fertility treatments, and an eventual increase in the waitlist for services once they resume. The extent of the impact will depend on the duration of service postponement and regional resource variations (e.g. access to operating theatre time when surgeries resume).

What management options are available during the COVID-19 pandemic?

We encourage individuals in need of help to seek a clinical assessment with their general practitioners (GP), 
gynaecologists, physiotherapists, and/or complementary medical providers through telehealth avenues. Referral to a gynaecologist with expertise in endometriosis may also be appropriate to offset the new diagnostic and therapeutic challenges faced during this time. Those with an established diagnosis who are seeking help, regardless of their intentions to pursue surgical management, should discuss with their clinician the potential to modify their current medication regimen. Some with suspected endometriosis may accept a clinical diagnosis in the absence of imaging or laparoscopy and empirical medical therapy can be initiated. ${ }^{8}$

Caution in the use of non-steroidal anti-inflammatory drugs (NSAIDs), commonly used for endometriosisrelated pain, is being advised, because increase in angiotensin-converting enzyme 2 (ACE2) may predispose to infection with COVID-19. ${ }^{9}$ Those at low-risk of exposure may consider the benefits of NSAIDs outweigh potential risk, deciding to continue use when needed. Conversely, those at higher-risk for acquisition (e.g. continuing to attend work outside of their home or having household members whom are high-risk), may benefit from using NSAIDs. Beyond traditional medical therapies, problem-focused interventions such as education, modifying work/school/social life, taking advantage of virtual and telephone support provided by national endometriosis organisations, improving sleep hygiene, low-intensity physical activity (including pelvic exercises, yoga), dietary changes, application of heat, and medical cannabis should be considered, either with the assistance of a healthcare provider via telehealth or independently by patients themselves. Similarly, emotion-focused strategies, which include relaxation/mindfulness, acceptance of chronic illness (e.g. via Acceptance and Commitment Therapy with the help of a clinical psychologist through telehealth), reducing catastrophising, and improving a balance toward positive attitude can be considered. These strategies are not unique to the COVID-19 pandemic and are recognised as an integral part of the usual multidisciplinary treatment of endometriosis.

Patients should be aware that, if they experience acute exacerbations of their chronic pain, they may warrant urgent medical assessment, as such cases, especially those with suspected endometrioma or severe acute recalcitrant exacerbation of pain, may require urgent surgery. However, most pain exacerbations are not lifeor organ-threatening and with appropriate counselling and support, a face-to-face consultation in the emergency department may be avoided. Some GPs may find it challenging to confidently reassure patients that they are safe to avoid an emergency department visit, so urgent telehealth consultation with a gynaecologist or pain specialist may be helpful.

What will the endometriosis community learn from the COVID-19 experience?

This pandemic has been, and remains to be, a very difficult period for individuals with endometriosis. However, this panel believes the consequences of the pandemic will yield a change in how clinical medicine for endometriosis is conducted. Firstly, there may be an ongoing openness to telehealth. This could dramatically minimise the geographic barriers to care that many women experience and facilitate the development of endometriosis networks of expertise, which is recommended by the World Endometriosis Society. ${ }^{8}$ Telehealth may also be an appropriate alternative for patients with pain that limits their ability to travel to their healthcare provider in some settings. Second, there may be increased awareness to self-management strategies that have always existed, yet were under-utilised. Finally, the current situation mandates a more discerning approach to surgery now and in the future, so that we 'operate sparingly and operate well'. This approach can be guided by preoperative triaging tools including advanced clinical algorithms and imaging strategies ${ }^{10}$ to avoid multiple repeated surgical procedures.

Box 1 Clinical guidance summary for managing patients with endometriosis during the COVID-19 pandemic and beyond

Be aware of potential endometriosis risk factors for COVID-19 infection that include Patients in whom endom

\section{Authors' Roles}

All authors meet justification criteria of authorship as per ICMJE: 
1. substantial contributions to conception and design, or acquisition of data, or analysis and interpretation of data,

2. drafting the article or revising it critically for important intellectual content,

3. final approval of the version to be published and

4. agreement to be accountable for all aspects of the work in ensuring that questions related to the accuracy or integrity of any part of the work are appropriately investigated and resolved.

\section{Acknowledgments}

None

\section{Funding Statement}

None

\section{Conflict of Interest}

Dr. Leonardi reports grants from Australian Women and Children's Research Foundation, outside the submitted work. Prof. Horne reports grants from Chief Scientist Office, NIHR EME, MRC, Wellbeing of Women, Ferring, and Roche Diagnostics during the conduct of the study; and honoraria for consultancy for Ferring, Roche, and AbbVie, outside the submitted work. Dr. Armour reports grants from Metagenics and Spectrum outside the submitted work. Prof. Missmer reports a grant and consulting fees from Abbvie, and consulting fees from Roche outside the submitted work. Prof. Rombauts reports personal fees from Monash IVF Group, grants from Ferring Australia, personal fees from Ferring Australia, non-financial support from Merck Serono, non-financial support from MSD, non-financial support from Guerbet, outside the submitted work; and Minority shareholder and Group Medical Director for Monash IVF Group and the President-Elect of the World Endometriosis Society. Prof. Roman reports personal fees from Olympus, personal fees from Ethicon, personal fees from Nordic Pharma, personal fees from Plasma Surgical Ltd, outside the submitted work. Ms Hummelshoj reports personal fees from AbbVie, is the chief executive of the World Endometriosis Society and the owner of Endometriosis.org., outside the submitted work. Prof. Condous reports personal fees from Roche, personal fees from GE Healthcare, grants from Australian Women and Children's Research Foundation, outside the submitted work. Prof. Johnson reports personal fees from Guerbet, personal fees from Vifor Pharma, grants and personal fees from Myovant Sciences, grants from AbbVie, personal fees from Roche, outside the submitted work. Prof. Wattiez has nothing to disclose.

\section{References}

1. Johnson NP, Hummelshoj L, Adamson GD, Keckstein J, Taylor HS, Abrao MS, et al. World endometriosis society consensus on the classification of endometriosis. Hum Reprod . 2017;32:315-24.

2. Zondervan KT, Becker CM, Missmer SA. Endometriosis. Longo DL, editor.N Engl J Med . 2020 Mar $26 ; 382: 1244-56$.

3. Horne AW, Saunders PTK. SnapShot: Endometriosis. Cell . 2019 Dec;179:1677-1677.e1.

4. AAGL - Elevating Gynecologic Surgery. COVID-19: Joint Statement on Elective Surgeries [Internet]. 2020 [cited 2020 Apr 5]. Available from: https://www.aagl.org/news/covid-19-joint-statement-on-electivesurgeries/

5. American Society for Reproductive Medicine. COVID-19: Suggestions On Managing Patients Who Are Undergoing Infertility Therapy Or Desiring Pregnancy [Internet]. 2020 [cited 2020 Mar 24]. Available from: https://www.asrm.org/news-and-publications/news-and-research/press-releases-and-bulletins/covid-19-suggestionson-managing-patients-who-are-undergoing-infertility-therapy-or-desiring-pregnancy/

6. Bourne T, Leonardi M, Kyriacou C, Al-Memar M, Landolfo C, Cibula D, et al. ISUOG Consensus Statement on rationalization of gynecological ultrasound services in context of SARS-CoV-2. Ultrasound Obstet Gynecol . 2020;10.1002/uog.22047. 
7. People Who Are at Higher Risk for Severe Illness | Coronavirus | COVID-19 | CDC [Internet]. [cited 2020 Apr 18]. Available from: https://www.cdc.gov/coronavirus/2019-ncov/need-extra-precautions/groupsat-higher-risk.html

8. Johnson NP, Hummelshoj L, World Endometriosis Society Montpellier Consortium. Consensus on current management of endometriosis. Hum Reprod . 2013 Jun 1;28:1552-68.

9. Fang L, Karakiulakis G, Roth M. Are patients with hypertension and diabetes mellitus at increased risk for COVID-19 infection? Lancet Respir Med . 2020 Apr;8:e21.

10. Leonardi M, Espada M, Choi S, Chou D, Chang T, Smith C, et al. Transvaginal Ultrasound Can Accurately Predict the American Society of Reproductive Medicine Stage of Endometriosis Assigned at Laparoscopy.J Minim Invasive Gynecol . 2020 Feb 22;10.1016/j.jmig.2020.02.014. 